\title{
Interactions of unparticles with standard model particles
}

\author{
Shao-Long Chen* and Xiao-Gang $\mathrm{He}^{\dagger}$ \\ Department of Physics and Center for Theoretical Sciences, National Taiwan University, Taipei, Taiwan
}

(Received 5 June 2007; published 16 November 2007)

\begin{abstract}
We study interactions of unparticles $\mathcal{U}$ of dimension $d \mathcal{u}$ due to Georgi with standard model (SM) fields through effective operators. The unparticles describe the low energy physics of a nontrivial scale invariant sector. Since unparticles come from beyond the SM physics, it is plausible that they transform as a singlet under the SM gauge group. This helps tremendously in limiting possible interactions. We analyze interactions of scalar $\mathcal{U}$, vector $\mathcal{U}^{\mu}$, and spinor $\mathcal{U}^{s}$ unparticles with SM fields and derivatives up to dimension four. Using these operators, we discuss different features of producing unparticles at an $e^{+} e^{-}$ collider and other phenomenologies. It is possible to distinguish different unparticles produced at an $e^{+} e^{-}$ collider by looking at various distributions of production cross sections.
\end{abstract}

DOI: 10.1103/PhysRevD.76.091702

PACS numbers: 12.60.-i, 13.66.Hk, 13.90.+i

In a scale invariant theory in four space-time dimensions, there are no particles with a nonzero mass. In our real world, there are plenty of particles with nonzero masses. If scale invariance plays a role in nature, it must have been broken at some high energy scale beyond the standard model (SM) scale. Recently Georgi proposed an interesting idea to describe a possible scale invariant effect at low energies, termed unparticle [1]. Based on a specific model scale invariant theory by Banks and Zaks (BZ) [2], Georgi argued that operators $O_{\mathrm{BZ}}$ made of BZ fields may interact with operators $O_{\mathrm{SM}}$ made of SM fields at some high energy scale by exchange particles with large masses, $M_{u}$, with the generic form $O_{\mathrm{SM}} O_{\mathrm{BZ}} / M_{u}^{k}$. At another scale $\Lambda_{u}$ the BZ sector induces dimensional transmutation; below that scale the $\mathrm{BZ}$ operator $O_{\mathrm{BZ}}$ matches onto the unparticle operator $O_{u}$ with dimension $d_{u}$, and the unparticle interaction with SM particles at low energy has the form $\lambda \Lambda_{u}^{4-d_{\mathrm{SM}}-d_{u}} O_{\mathrm{SM}} O_{u}$. Here $d_{\mathrm{SM}}$ is the dimension of the operator $O_{\mathrm{SM}}$.

An unparticle looks like a nonintegral $d_{u}$ dimension invisible particle. Depending on the nature of the original operator $O_{\mathrm{BZ}}$ and the transmutation, the resulting unparticles may have different Lorentz structure. We will indicate an unparticle that acts like a Lorentz scalar as $O_{\mathcal{U}}$, a vector as $O_{u}^{\mu}$, and a spinor as $O_{U}^{s}$.

For detailed studies, one also needs to know how an unparticle interacts with SM particles. Recent studies have focused on several low dimension operators [1,3-16]. The unparticle interactions with the SM particles are through exchange of some other heavy particles of mass $M u$. Therefore the form of the interaction is basically determined by the nature of the heavy particle. If it is an SM singlet, the unparticle $O_{\mathcal{U}}$ resulting from the transmutation should also transform under the SM gauge group as a singlet. One cannot rule out other possibilities. There are many ways that the SM fields can couple to an unparticle. If the unparticle is an SM singlet, the possibilities are limited since the SM fields have to form an SM singlet first. In this work we concentrate on possible interactions of unparticles with the SM particles assuming that unparticles transform as SM singlets, and study some implications.

In the following we list operators composed of SM fields and derivatives with dimensions less than or equal to 4 invariant under the SM gauge group.

Scalar $O_{u}$ couplings:

(a) Couplings with gauge bosons

$$
\begin{array}{lc}
\lambda_{g g} \Lambda_{u}^{-d u} G^{\mu \nu} G_{\mu \nu} O_{u}, & \lambda_{w w} \Lambda_{u}^{-d u} W^{\mu \nu} W_{\mu \nu} O_{u}, \\
\lambda_{b b} \Lambda_{u}^{-d u} B^{\mu \nu} B_{\mu \nu} O_{u}, & \tilde{\lambda}_{g g} \Lambda_{u}^{-d u} \tilde{G}^{\mu \nu} G_{\mu \nu} O_{u}, \\
\tilde{\lambda}_{w w} \Lambda_{u}^{-d u} \tilde{W}^{\mu \nu} W_{\mu \nu} O_{u}, & \tilde{\lambda}_{b b} \Lambda_{u}^{-d_{u}} \tilde{B}^{\mu \nu} B_{\mu \nu} O_{u},
\end{array}
$$

(b) Coupling with Higgs and Gauge bosons

$$
\begin{aligned}
& \lambda_{h h} \Lambda_{u}^{2-d_{u}} H^{\dagger} H O_{u}, \\
& \tilde{\lambda}_{h h} \Lambda_{u}^{-d_{u}}\left(H^{\dagger} D_{\mu} H\right) \partial^{\mu} O_{u}, \\
& \lambda_{4 h} \Lambda_{\mathcal{U}}^{-d_{u}}\left(H^{\dagger} H\right)^{2} O_{u}, \\
& \lambda_{d h} \Lambda_{\mathcal{U}}^{-d_{u}}\left(D_{\mu} H\right)^{\dagger}\left(D^{\mu} H\right) O_{u},
\end{aligned}
$$

(c) Couplings with fermions and gauge bosons

\footnotetext{
*shaolong@phys.ntu.edu.tw

†hexg@phys.ntu.edu.tw
} 


$$
\begin{aligned}
& \lambda_{Q Q} \Lambda_{\mathcal{U}}^{-d u} \bar{Q}_{L} \gamma_{\mu} D^{\mu} Q_{L} O_{\mathcal{U}}, \\
& \lambda_{U U} \Lambda_{U}^{-d u} \bar{U}_{R} \gamma_{\mu} D^{\mu} U_{R} O_{\mathcal{U}} \text {, } \\
& \lambda_{D D} \Lambda_{U}^{-d u} \bar{D}_{R} \gamma_{\mu} D^{\mu} D_{R} O_{\mathcal{U}} \text {, } \\
& \lambda_{L L} \Lambda_{\mathcal{U}}^{-d_{u}} \bar{L}_{L} \gamma_{\mu} D^{\mu} L_{L} O_{\mathcal{U}}, \\
& \lambda_{E E} \Lambda_{\mathcal{U}}^{-d_{u}} \bar{E}_{R} \gamma_{\mu} D^{\mu} E_{R} O_{\mathcal{U}}, \\
& \lambda_{\nu \nu} \Lambda_{\mathcal{U}}^{-d}{ }^{d} \bar{\nu}_{R} \gamma_{\mu} D^{\mu} \nu_{R} O_{\mathcal{U}} \text {, } \\
& \tilde{\lambda}_{Q Q} \Lambda_{\mathcal{U}}^{-d u} \bar{Q}_{L} \gamma_{\mu} Q_{L} \partial^{\mu} O_{\mathcal{U}} \text {, } \\
& \tilde{\lambda}_{U U} \Lambda_{\mathcal{U}}^{-d} \bar{U}_{R} \gamma_{\mu} U_{R} \partial^{\mu} O_{\mathcal{U}} \text {, } \\
& \tilde{\lambda}_{D D} \Lambda_{\mathcal{U}}^{-d u} \bar{D}_{R} \gamma_{\mu} D_{R} \partial^{\mu} O_{\mathcal{U}} \text {, } \\
& \tilde{\lambda}_{L L} \Lambda_{\mathcal{U}}^{-d u} \bar{L}_{L} \gamma_{\mu} L_{L} \partial^{\mu} O_{\mathcal{U}} \text {, } \\
& \tilde{\lambda}_{E E} \Lambda_{\mathcal{U}}^{-d u} \bar{E}_{R} \gamma_{\mu} E_{R} \partial^{\mu} O_{\mathcal{U}} \text {, } \\
& \tilde{\lambda}_{R R} \Lambda_{\mathcal{U}}^{-d} \bar{\nu}_{R} \gamma_{\mu} \nu_{R} \partial^{\mu} O_{\mathcal{U}} \text {, } \\
& \lambda_{Y R} \Lambda_{\mathcal{U}}^{1-d_{u}} \bar{\nu}_{R}^{C} \nu_{R} O_{\mathcal{U}},
\end{aligned}
$$

(d) Couplings with fermions and Higgs boson

$$
\begin{array}{lc}
\lambda_{Y U} \Lambda_{\mathcal{U}}^{-d u} \bar{Q}_{L} H U_{R} O_{\mathcal{U}}, & \lambda_{Y D} \Lambda_{u}^{-d u} \bar{Q}_{L} \tilde{H} D_{R} O_{u}, \\
\lambda_{Y \nu} \Lambda_{u}^{-d} \bar{L}_{L} H \nu_{R} O_{u}, & \lambda_{Y E} \Lambda_{\mathcal{u}}^{-d_{u}} \bar{L}_{L} \tilde{H} E_{R} O_{u} .
\end{array}
$$

Vector $O_{u}^{\mu}$ couplings:

(a) Couplings with fermions

$$
\begin{aligned}
& \lambda_{Q Q}^{\prime} \Lambda_{\mathcal{U}}^{1-d u} \bar{Q}_{L} \gamma_{\mu} Q_{L} O_{\mathcal{U}}^{\mu}, \\
& \lambda_{U U}^{\prime} \Lambda_{\mathcal{U}}^{1-d u} \bar{U}_{R} \gamma_{\mu} U_{R} O_{\mathcal{u}}^{\mu}, \\
& \lambda_{D D}^{\prime} \Lambda_{\mathcal{U}}^{1-d u} \bar{D}_{R} \gamma_{\mu} D_{R} O_{\mathcal{u}}^{\mu}, \\
& \lambda_{L L}^{\prime} \Lambda_{\mathcal{U}}^{1-d u} \bar{L}_{L} \gamma_{\mu} L_{L} O_{\mathcal{u}}^{\mu}, \\
& \lambda_{E E}^{\prime} \Lambda_{\mathcal{U}}^{1-d u} \bar{E}_{R} \gamma_{\mu} E_{R} O_{\mathcal{U}}^{\mu}, \\
& \lambda_{R R}^{\prime} \Lambda_{\mathcal{U}}^{1-d u} \bar{\nu}_{R} \gamma_{\mu} \nu_{R} O_{\mathcal{U}}^{\mu},
\end{aligned}
$$

(b) Couplings with Higgs boson and Gauge bosons

$$
\lambda_{h h}^{\prime} \Lambda_{\mathcal{U}}^{1-d_{u}}\left(H^{\dagger} D_{\mu} H\right) O_{\mathcal{U}}^{\mu}, \quad \lambda_{b O}^{\prime} \Lambda_{\mathcal{u}}^{1-d_{u}} B_{\mu \nu} \partial^{\mu} O^{\nu} .
$$

Spinor $O_{U}^{s}$ couplings:

$$
\lambda_{s \nu} \Lambda_{\mathcal{U}}^{5 / 2-d u} \bar{\nu}_{R} O_{u}^{s}, \quad \lambda_{s} \Lambda_{U}^{3 / 2-d}{ }^{2} \bar{L}_{L} H O_{u}^{s} .
$$

Here $G, W$, and $B$ are the $S U(3)_{C}, S U(2)_{L}$, and $U(1)_{Y}$ gauge fields, respectively. $Q_{L}, U_{R}, D_{R}, L_{L}, E_{R}$ are the SM left-handed quark doublet, right-handed up quark, righthanded down quark, left-handed lepton doublet, and righthanded charged lepton, respectively. In the above we also included the right-handed neutrino $\nu_{R}$ which might be needed from neutrino oscillation data.

The scalar $\mathcal{U}$ unparticle has the largest number of operators. In this class of interactions, the lowest SM dimension operators is the coupling of $U$ to two Higgs fields, $\mathrm{H}^{\dagger} H \mathrm{O}_{U}$. The second lowest operator involves two right-handed neutrinos, $\bar{\nu}_{R}^{C} \nu_{R} O_{u}$. The rest have the same dimensions with the SM fields and derivatives forming dimension four operators.

The operator $H^{\dagger} H O_{u}$ with a low dimension $\Lambda_{u}^{2-d u}$ may have the best chance to show up at low energies. An effect is that when the Higgs field develops a nonzero vacuum expectation value (VEV) $\langle H\rangle=v / \sqrt{2}$ as required by gauge symmetry breaking and generation of SM particle masses, there is a tadpole coupling $\lambda_{h h} \Lambda_{u}^{2-d_{u}} v^{2} / 2$ of unparticle to VEV which introduces a scale to the unparticle sector. This interaction will cause the unparticle sector to be pushed away from its scale invariant fixed point and the theory become nonscale invariant at some low scale. Below that the unparticle sector presumably becomes a traditional particle sector [13]. We note that this may not be necessarily true if one also includes the other operator $\left(H^{\dagger} H\right)^{2} \mathrm{O} u$. This term also has a tadpole coupling of unparticle to VEV. It is given by $\lambda_{4 h} \Lambda_{u}^{-d}$. If $\lambda_{h h} \Lambda_{u}^{2}+$ $\lambda_{4 h} v^{2} / 2=0$, the tadpole will be removed. One then has

$$
\begin{aligned}
& \lambda_{h h} \Lambda_{u}^{2-d_{u}} H^{\dagger} H O_{u}+\lambda_{4 h} \Lambda_{u}^{-d_{u}}\left(H^{\dagger} H\right)^{2} O_{u} \\
& \quad=\frac{1}{4} \lambda_{4 h} \Lambda_{u}^{-d_{u}}\left(h^{4}+4 v h^{3}+5 v^{2} h^{2}+2 v^{3} h\right) O_{u} .
\end{aligned}
$$

Here, we have removed the would-be Goldstone boson in the Higgs field, and $h$ is the physical Higgs field. The operators above will induce mixing between $h$ and the scalar unparticle. We should note that the cancellation mechanism discussed above is by assumption. We are not able to find a symmetry to guarantee it, and it may not be stable. Another possibility is that these couplings cannot be generated such that the unparticle physics effect can still show up at low energies. More studies are needed.

The operator $\bar{\nu}_{R}^{C} \nu_{R} O_{U}$ involves a right-handed neutrino $\nu_{R}$ interaction with an unparticle. If $\nu_{R}$ is heavy, there is no observable effect. If $\nu_{R}$ turns out to be a light sterile neutrino, one may see some effects in neutrino decays; a heavier $\nu_{R}$ may decay into a lighter $\nu_{R}$ and $O_{U}$.

There are six operators involving $O_{U}$ and gauge particles. The interactions with gluon fields can produce $O_{U}$ at hadron colliders through $g g \rightarrow g \mathcal{U}, q \bar{q} \rightarrow g \mathcal{U}$, and $g q \rightarrow$ $q \mathcal{U}$. The operators with $W$ and $B$ can produce $\mathcal{U}$ at a photon collider through $\gamma \gamma \rightarrow \mathcal{U}, \gamma e \rightarrow \gamma \mathcal{U}$, and also an interesting signature in $W W$ scattering [14].

The operators in class (c) have rich phenomenology. Several of the operators have been studied in flavor changing decay of a heavy fermion to a light fermion plus an unparticle such as $t \rightarrow u(c)+\mathcal{U}$, meson and antimeson mixing, and other flavor changing decays. These operators can also produce $\mathcal{U}$ at hadron and $e^{+} e^{-}$colliders.

The operators in class (d) involve an unparticle with SM Yukawa terms [13]. These will open new decay channels for the Higgs and the top quark with an unparticle in the final state. They can also induce $e^{+} e^{-}(q \bar{q}) \rightarrow h \mathcal{U}$. 


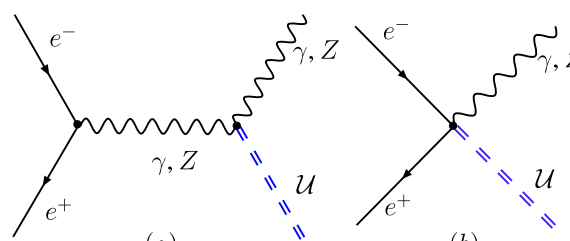

(a) (b)

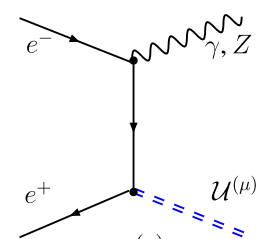

(c)

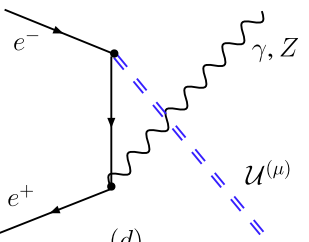

(d)

FIG. 1 (color online). The Feynman diagrams for $\mathcal{U}$ and $\mathcal{U}^{\mu}$ productions through $e^{+} e^{-} \rightarrow \gamma(Z)+\mathcal{U}^{(\mu)}$. For $\mathcal{U}^{\mu}$ production, only diagrams (c) and (d) contribute.

There are less operators involving the vector $\mathcal{U}^{\mu}$ and an SM particle. The class (a) operators are the most studied ones. Similar to class (c) operators for scalar unparticle couplings, they can induce $t \rightarrow u(c)+\mathcal{U}$, meson and antimeson mixing, and can also produce $\mathcal{U}$ at hadron and $e^{+} e^{-}$colliders. The first operator in class (b) can induce $h$ and unparticle mixing, and the second operator can induce $B$ and unparticle mixing.

The unparticle may have spinor structure under the Lorentz group [5]. There are only two operators for spinor unparticle and SM particle interactions. The operator $\bar{\nu}_{R} O_{U}^{s}$ has the lowest dimension in the whole list. This operator will mix the unparticle with a right-handed neutrino. Deviations of the neutrino oscillation pattern may be the best place for looking for unparticle effects. The operator $\bar{L}_{L} H O_{U}^{s}$ can induce mixing between a left-handed neutrino and an unparticle. Again this will affect neutrino mixing and also cause the mixing matrix to be not the usual $3 \times 3$ unitary matrix for three left-handed neutrinos. This operator will cause the Higgs to decay into a neutrino and an unparticle.

There is a lot of interesting phenomenology which can be carried out using the above listed interactions. Besides the production of unparticles, there are also virtual effects of unparticles [1,3-16]. In the rest of the paper we concentrate on the possibility of distinguishing whether an $O_{\mathcal{U}}$ or an $O_{u}^{\mu}$ is produced through $e^{+} e^{-}$collisions through $e^{+} e^{-} \rightarrow \gamma(Z)+\mathcal{U}, \gamma(Z)+\mathcal{U}^{\mu}$.

The Feynman diagrams for the above processes are shown in Fig. 1. For $\mathcal{U}$ production, the operators

$$
\begin{aligned}
& |\overline{\mathcal{M}}|^{2}\left(e^{+} e^{-} \rightarrow \gamma \mathcal{U}\right)=\frac{e^{2} s\left(u^{2}+t^{2}\right)}{\Lambda_{u}^{2 d u}} f\left(\lambda_{w w}, \lambda_{b b}\right), \\
& |\overline{\mathcal{M}}|^{2}\left(e^{+} e^{-} \rightarrow Z \mathcal{U}\right)=\frac{e^{2} s\left[\left(t-m_{Z}^{2}\right)^{2}+\left(u-m_{Z}^{2}\right)^{2}+2 s m_{Z}^{2}\right]}{\Lambda_{\mathcal{U}}^{2 d u}} g\left(\lambda_{w w}, \lambda_{b b}\right), \\
& |\overline{\mathcal{M}}|^{2}\left(e^{+} e^{-} \rightarrow Z \mathcal{U}\right)=\frac{e^{2} s\left[\left(t-m_{Z}^{2}\right)^{2}+\left(u-m_{Z}^{2}\right)^{2}-2 s m_{Z}^{2}\right]}{\Lambda_{U}^{2 d u}} g\left(\tilde{\lambda}_{w w}, \tilde{\lambda}_{b b}\right), \\
& |\overline{\mathcal{M}}|^{2}\left(e^{+} e^{-} \rightarrow \gamma \mathcal{U}\right)=\frac{2 e^{2}\left(\lambda_{L L}^{2}+\lambda_{E E}^{2}\right)}{\left(\Lambda_{U}^{2}\right)^{d u^{-1}}} \frac{s}{\Lambda_{U}^{2}}, \quad|\overline{\mathcal{M}}|^{2}\left(e^{+} e^{-} \rightarrow \gamma \mathcal{U}^{\mu}\right)=\frac{e^{2}\left(\lambda_{L L}^{\prime 2}+\lambda_{E E}^{\prime 2}\right)}{\left(\Lambda_{U}^{2}\right)^{d u^{-1}}} \frac{u^{2}+t^{2}+2 s P_{u}^{2}}{u t}, \\
& |\overline{\mathcal{M}}|^{2}\left(e^{+} e^{-} \rightarrow Z \mathcal{U}\right)=\frac{2 g^{2}}{\cos ^{2} \theta_{w}} \frac{\left(\lambda_{L L}^{2}\left(\frac{1}{2}-\sin ^{2} \theta_{w}\right)^{2}+\lambda_{E E}^{2} \sin ^{4} \theta_{w}\right)}{\left(\Lambda_{U}^{2}\right)^{d} u^{-1}} \frac{\left(s+\frac{u t}{2 m_{Z}^{2}}\right)}{\Lambda_{u}^{2}}, \\
& |\overline{\mathcal{M}}|^{2}\left(e^{+} e^{-} \rightarrow Z \mathcal{U}^{\mu}\right)=\frac{g^{2}}{\cos ^{2} \theta_{w}} \frac{\left(\lambda_{L L}^{\prime 2}\left(\frac{1}{2}-\sin ^{2} \theta_{w}\right)^{2}+\lambda_{E E}^{\prime 2} \sin ^{4} \theta_{w}\right)}{\left(\Lambda_{U}^{2}\right)^{d u^{-1}}} \frac{\left(u^{2}+t^{2}+2 s\left(P_{u}^{2}+m_{Z}^{2}\right)-P_{u}^{2} m_{Z}^{2}\left(\frac{u}{t}+\frac{t}{u}\right)\right)}{u t},
\end{aligned}
$$


where

$$
\begin{aligned}
f\left(\lambda_{w w}, \lambda_{b b}\right)= & \frac{\left(\lambda_{w w} \sin ^{2} \theta_{w}+\lambda_{b b} \cos ^{2} \theta_{w}\right)^{2}}{s^{2}}+\frac{\left(\lambda_{w w}-\lambda_{b b}\right)^{2} 2\left[\left(\frac{1}{2}-\sin ^{2} \theta_{w}\right)^{2}+\sin ^{4} \theta_{w}\right]}{\left(s-m_{Z}^{2}\right)^{2}} \\
& +\frac{\left(\lambda_{w w} \sin ^{2} \theta_{w}+\lambda_{b b} \cos ^{2} \theta_{w}\right)\left(\lambda_{w w}-\lambda_{b b}\right)\left(1-4 \sin ^{2} \theta_{w}\right)}{s\left(s-m_{Z}^{2}\right)}, \\
g\left(\lambda_{w w}, \lambda_{b b}\right)= & \frac{\left(\lambda_{w w}-\lambda_{b b}\right)^{2} \sin ^{2} 2 \theta_{w}}{s^{2}}+\frac{\left(\lambda_{w w} \cot \theta_{w}+\lambda_{b b} \tan \theta_{w}\right)^{2}\left[\left(\frac{1}{2}-\sin ^{2} \theta_{w}\right)^{2}+\sin ^{4} \theta_{w}\right]}{2\left(s-m_{Z}^{2}\right)^{2}} \\
& +\frac{\left(\lambda_{w w}-\lambda_{b b}\right)\left(\lambda_{w w} \cos ^{2} \theta_{w}+\lambda_{b b} \sin ^{2} \theta_{w}\right)\left(1-4 \sin ^{2} \theta_{w}\right)}{s\left(s-m_{Z}^{2}\right)} .
\end{aligned}
$$

We now study the possibility of distinguishing $\mathcal{U}$ and $U^{\mu}$ productions. The photon and $Z$ boson angular and energy distributions are plotted taking $d_{\mathcal{U}}=1.5$ and $\sqrt{s}=$ $200 \mathrm{GeV}$, in Fig. 2, relevant for CERN LEP II data, and also $500 \mathrm{GeV}$, in Fig. 3, relevant for ILC for illustration. In both Figs. 2 and 3, we plot the distributions with different couplings setting others to zero. Since the electron mass is small, the cross section diverges at $\cos \theta= \pm 1$ for $\mathcal{U}^{\mu}$ production due to $u$ and $t$ appearing in the denominators if electron mass is neglected, but finite for $\mathcal{U}$ production. This provides a clear way to distinguish $\mathcal{U}$ and $\mathcal{U}^{\mu}$ production as can be seen from Figs. 2 and 3. The energy distributions can also provide useful information which can be seen from Figs. 2 and 3. For this case we made a cut with $\left|\cos \theta_{\gamma, Z}\right|<0.97$ to avoid the divergence for $\mathcal{U}^{\mu}$ production at $\left|\cos \theta_{\gamma, Z}\right|=1$.

The above discussion shows that the study of various distributions may be able to provide information about the type of unparticles at ILC if a large number of events can
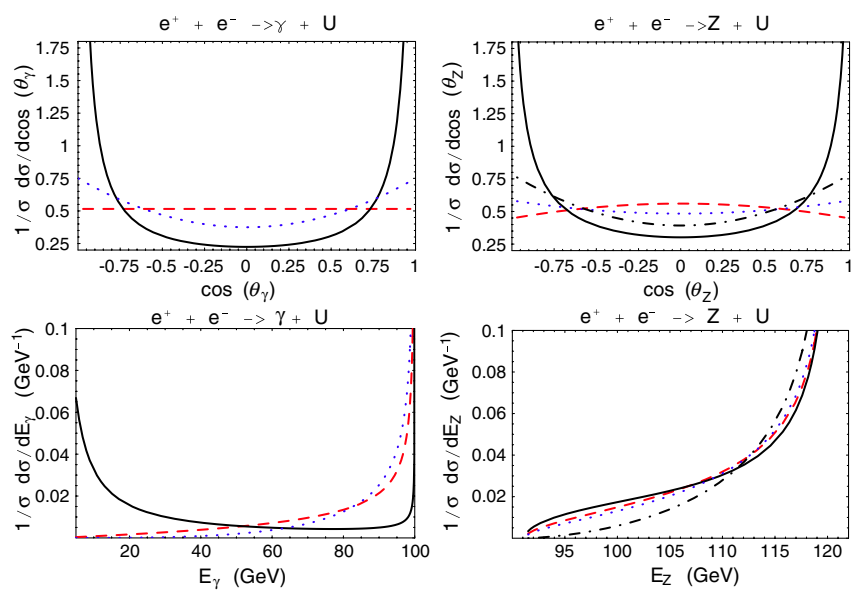

FIG. 2 (color online). Normalized photon ( $Z$ boson) energy spectrum and angular distribution of $e^{+} e^{-} \rightarrow \gamma(Z)+\mathcal{U}$ for $d_{u}=1.5$ at $\sqrt{s}=200 \mathrm{GeV}$. Dashed, solid, dotted, and dotdashed curves represent the contributions from the operators with couplings $\lambda_{L L, E E}\left(\lambda_{L L}\right.$ and $\lambda_{E E}$ give the same distributions), $\lambda_{L L, E E}^{\prime}\left(\lambda_{L L}^{\prime}\right.$ and $\lambda_{E E}^{\prime}$ give the same distributions), $\lambda_{w w, b b}$, and $\tilde{\lambda}_{w w, b b}$, respectively. Note that for the left panel, the curves are identical for the contributions from $\lambda_{w w, b b}, \tilde{\lambda}_{w w, b b}$. We have imposed $\left|\cos \theta_{\gamma, Z}\right|<0.97$ and $E_{\gamma}>5 \mathrm{GeV}$. be obtained. One needs, however, to see if existing constraints have already ruled out such possibilities. We now comment on constraints on the relevant coupling of the operators.

Presently there is a direct constraint from LEP II data [17] at $\sqrt{s}=207 \mathrm{GeV}$ on the cross section for $e^{+} e^{-} \rightarrow$ $\gamma X^{0}$ where $X^{0}$ is invisible. With the cuts $E_{\gamma}>5 \mathrm{GeV}$ and $|\cos \theta|<0.97$, the cross section $\sigma$ is constrained to be $\lesssim$ $0.2 \mathrm{pb}$ at $95 \%$ C.L. Interpreting $X^{0}$ as an unparticle, bounds can be obtained for combinations of parameters $\lambda_{i}$ and $\Lambda_{u}$. The corresponding bounds on various cross sections we are interested in can then be obtained. We list them in Table I for $d u=1.5$.

No observation of $e^{+} e^{-} \rightarrow \gamma+\mathcal{U}$ at LEP II may be due to too low event number. With larger integrated luminosity at ILC, unparticle effects may be observed. If the scale of unparticle physics is close to the upper bound, with an integrated luminosity of $100 \mathrm{fb}^{-1}$, the event numbers can reach more than $1.93 \times 10^{4}\left(1.3 \times 10^{3}\right)$ and $1.07 \times 10^{4}$ $\left(6.19 \times 10^{2}\right)$ for $e^{+} e^{-} \rightarrow \gamma(Z)+\mathcal{U}$ with $\sqrt{s}=200 \mathrm{GeV}$ and $\sqrt{s}=500 \mathrm{GeV}$, respectively. With an integrated luminosity of $500 \mathrm{fb}^{-1}$, the event numbers would be 5 times larger.

There are also several noncollider laboratory constraints directly related to the operators that have been studied


FIG. 3 (color online). Normalized photon ( $Z$ boson) energy spectrum and angular distribution of $e^{+} e^{-} \rightarrow \gamma(Z)+\mathcal{U}$ for $d_{\mathcal{u}}=1.5$ at $\sqrt{s}=500 \mathrm{GeV}$. 
TABLE I. Bounds on the cross sections using LEP II constraints with the cuts $|\cos \theta|<0.97$ and $E_{\gamma}>5$ GeV. Event numbers can be obtained by multiplying a given integrated luminosity.

\begin{tabular}{lcccc}
\hline \hline & \multicolumn{2}{c}{$\sqrt{s}=200 \mathrm{GeV}$} & \multicolumn{2}{c}{$\sqrt{s}=500 \mathrm{GeV}$} \\
$d_{\mathcal{U}}=1.5$ & $\sigma\left(e^{+} e^{-} \rightarrow \gamma \mathcal{U}\right)$ & $\sigma\left(e^{+} e^{-} \rightarrow Z \mathcal{U}\right)$ & $\sigma\left(e^{+} e^{-} \rightarrow \gamma \mathcal{U}\right)$ & $0.483 \mathrm{pb}$ \\
\hline$\lambda_{L L}$-term & $0.193 \mathrm{pb}$ & $0.0410 \mathrm{pb}$ & $0.483 \mathrm{pb}$ & $0.482 \mathrm{pb}$ \\
$\lambda_{E E^{-} \text {term }}$ & $0.193 \mathrm{pb}$ & $0.0266 \mathrm{pb}$ & $0.107 \mathrm{pb}$ & $0.313 \mathrm{pb}$ \\
$\lambda_{L L}^{\prime}$-term & $0.204 \mathrm{pb}$ & $0.0133 \mathrm{pb}$ & $0.107 \mathrm{pb}$ & $0.00954 \mathrm{pb}$ \\
$\lambda_{E E^{-}}^{\prime}-$ term & $0.204 \mathrm{pb}$ & $0.00863 \mathrm{pb}$ & $0.365 \mathrm{pb}$ & $0.00619 \mathrm{pb}$ \\
$\lambda_{w w}$-term & $0.198 \mathrm{pb}$ & $0.369 \mathrm{pb}$ & $0.365 \mathrm{pb}$ & $0.809 \mathrm{pb}$ \\
$\tilde{\lambda}_{w w}$-term & $0.198 \mathrm{pb}$ & $0.0969 \mathrm{pb}$ & $0.434 \mathrm{pb}$ & $0.616 \mathrm{pb}$ \\
$\lambda_{b b}$-term & $0.195 \mathrm{pb}$ & $0.162 \mathrm{pb}$ & $0.434 \mathrm{pb}$ & $0.355 \mathrm{pb}$ \\
$\tilde{\lambda}_{b b}$-term & $0.195 \mathrm{pb}$ & $0.0426 \mathrm{pb}$ & $0.270 \mathrm{pb}$ \\
\hline \hline
\end{tabular}

including $g-2$ of electron $[4,5,8]$ and invisible positronium decays [8]. We find that positronium decays into an $\mathcal{U}$ may provide a stronger bound than that at LEP II.

The operators with couplings $\lambda_{L L, E E}$ and $\lambda_{L L, E E}^{\prime}$ contribute to para-positronium to unparticle $(\mathrm{p}-\mathrm{Ps} \rightarrow \mathcal{U})$ ) and ortho-positronium to unparticle (o-Ps $\rightarrow \mathcal{U}$ ) decays directly at the tree level, respectively. The $90 \%$ C.L. experimental bounds on these decay branching ratios [18], $4.3 \times 10^{-7}$ and $4.2 \times 10^{-7}$, then lead to [19]

$$
\begin{aligned}
& A_{d_{u}}\left|\lambda_{L L}-\lambda_{E E}\right|^{2}\left(2 m_{e} / \Lambda_{U}\right)^{2 d_{u}<9.2 \times 10^{-9},} \\
& A_{d_{u}}\left|\lambda_{L L}^{\prime}+\lambda_{E E}^{\prime}\right|^{2}\left(2 m_{e} / \Lambda_{U}\right)^{2\left(d_{u}-1\right)}<2.0 \times 10^{-12} .
\end{aligned}
$$

In the above, our bound on scalar unparticle scale is different than that obtained in Ref. [8] is due to the fact that we have required the unparticles to couple to SM invariant operators such that the couplings scale as $\Lambda_{u}^{-2 d u}$ while in Ref. [8] they scale as $\Lambda_{u}^{-2\left(d_{u}-1\right)}$.

The constraints on $\lambda_{L L, E E}$ are much weaker than that from LEP II. The constraints on $\lambda_{L L, E E}^{\prime}$ are, however, much stronger. Assuming no cancellation between $\lambda_{L L}^{\prime}$ and $\lambda_{E E}^{\prime}$, the bounds would imply that the cross sections for $e^{+} e^{-} \rightarrow$ $\gamma(Z)+\mathcal{U}$ to be less than $5.5 \times 10^{-5}\left(3.4 \times 10^{-6}\right) \mathrm{pb}$ at $\sqrt{s}=200 \mathrm{GeV}$ and $2.9 \times 10^{-5}\left(2.3 \times 10^{-6}\right) \mathrm{pb}$ at $\sqrt{s}=$ $500 \mathrm{GeV}$ for $d_{\mathcal{U}}=1.5$. If true, the unparticle effects due to these operators will not be able to be studied at ILC. However, with larger $d_{u}$, it is still possible. For example, for $d_{u}=1.88$, one can get more than $4.6 \times 10^{4}(1.2 \times$ $\left.10^{3}\right)$ and $5.1 \times 10^{4}\left(2.6 \times 10^{3}\right) e^{+} e^{-} \rightarrow \gamma(Z)+\mathcal{U}$ events at $\sqrt{s}=200 \mathrm{GeV}$ and $500 \mathrm{GeV}$, respectively, with the integrated luminosity of $100 \mathrm{fb}^{-1}$. We should also note that since the constraint is proportional to $\lambda_{L L}^{\prime}+\lambda_{E E}^{\prime}$, if there is a cancellation such that this quantity is small, but individual $\lambda_{L L, E E}^{\prime}$ is not small, the cross sections for $e^{+} e^{-} \rightarrow \gamma(Z)+\mathcal{U}$ can still be large and unparticle physics effects can still be studied at ILC.

No constraints on $\lambda_{w w, b b}\left(\tilde{\lambda}_{w w, b b}\right)$ can be obtained from a positronium decay into an unparticle. However, one can obtain constraints from o-Ps $\rightarrow \gamma+\mathcal{U}$ decay through the diagrams shown in Fig. 1. We have carried out such a study using formulas in Ref. [20] for $\Upsilon \rightarrow \gamma+\mathcal{U}$ with appropriate replacement for parameters. We find that the constraints are much weaker than that from LEP II data.

The work of the authors was supported in part by the NSC and NCTS.
[1] H. Georgi, Phys. Rev. Lett. 98, 221601 (2007).

[2] T. Banks and A. Zaks, Nucl. Phys. B206, 23 (1982).

[3] H. Georgi, Phys. Lett. B 650, 275 (2007).

[4] K. Cheung et al., arXiv:0704.2588.

[5] M. Luo and G. Zhu, arXiv:0704.3532.

[6] C. H. Chen and C. Q. Geng, arXiv:0705.0689.

[7] G. J. Ding and M. L. Yan, Phys. Rev. D 76, 075005 (2007).

[8] Y. Liao, Phys. Rev. D 76, 056006 (2007).

[9] T. M. Aliev et al., arXiv:0705.1326.

[10] X. Q. Li and Z. T. Wei, Phys. Lett. B 651, 380 (2007).

[11] C. D. Lu et al., Phys. Rev. D 76, 077701 (2007).

[12] M. A. Stephanov, Phys. Rev. D 76, 035008 (2007).

[13] P. J. Fox et al., Phys. Rev. D 76, 075004 (2007).
[14] N. Greiner, Phys. Lett. B 653, 75 (2007).

[15] H. Davoudiasl, Phys. Rev. Lett. 99, 141301 (2007).

[16] D. Choudhury et al., arXiv:0705.3637.

[17] A. Heister et al. (ALEPH Collaboration), Eur. Phys. J. C 28, 1 (2003); J. Abdallah et al. (DELPHI Collaboration), Eur. Phys. J. C 38, 395 (2005); P. Achard et al. (L3 Collaboration), Phys. Lett. B 587, 16 (2004); G. Abbiendi et al. (OPAL Collaboration), Eur. Phys. J. C 18, 253 (2000).

[18] A. Baderscher et al., Phys. Rev. D 75, 032004 (2007).

[19] We have corrected a missing factor of $3 / 2$ for $\Gamma(0-\mathrm{P} \rightarrow$ $\mathcal{U})$ in Ref. [8].

[20] S. L. Chen et al., arXiv:0707.0187. 